\title{
Sivers and Collins Effects: from SIDIS to Proton-Proton Inclusive Pion Production
}

\author{
M. Anselmino ${ }^{1,2}$, M. Boglione ${ }^{1,2}$, U. D’Alesio ${ }^{3,4}$, E. Leader ${ }^{5}$, S. Melis ${ }^{2,6}$, F. Murgia ${ }^{4}$, A. Prokudin ${ }^{1,2}$ \\ 1- Università di Torino - Dipartimento di Fisica Teorica - \\ Via P. Giuria 1, I-10125 Torino - Italy \\ 2- INFN - Sezione di Torino - \\ Via P. Giuria 1, I-10125 Torino - Italy \\ 3- Università di Cagliari - Dipartimento di Fisica - \\ C.P. 170, I-09042 Monserrato (CA) - Italy \\ 4- INFN - Sezione di Cagliari - \\ C.P. 170, I-09042 Monserrato (CA) - Italy \\ 5- Imperial College London- South Kensington Campus \\ Prince Consort Road, London SW7 2AZ - U.K. \\ 6- Università del Piemonte Orientale - \\ Dipartimento di Scienze e Tecnologie Avanzate - \\ Viale T. Michel 11, I-15121 Alessandria - Italy
}

\begin{abstract}
We consider the Sivers, Collins and transversity functions as extracted from SIDIS and $e^{+} e^{-}$experimental data and investigate to what extent they might explain the large Single Spin Asymmetries (SSA) observed in proton-proton inclusive processes. This phenomenological study is performed within the TMD factorization scheme. As the SIDIS data cover only a limited range of $x$ values $(x \lesssim 0.3)$, we allow for different large $x$ behaviours of the SIDIS Sivers functions and transversity distributions. We conclude that, within the available experimental constraints, one cannot observe any clear universality breaking effect for the Sivers functions.
\end{abstract}

We report [1] on some work in progress, exploring the simple phenomenological idea of adopting the same Sivers, Collins and transversity functions, as extracted from SIDIS and $e^{+} e^{-}$experimental data [2, 3, to evaluate the corresponding Sivers and Collins effects in proton-proton scattering, assuming a TMD factorized scheme. These effects are then compared to the RHIC proton-proton data on SSAs at $\sqrt{s}=200 \mathrm{GeV}$ [4, 5].

The $A_{U T}^{\text {Sivers }}$ transverse single spin asymmetry, measured by the HERMES [6, 7] and COMPASS [8, 9, 10, 11] collaborations in $\ell N \rightarrow \ell h X$ SIDIS processes, has been analyzed according to the expression:

$$
A_{U T}^{\text {Sivers }} \propto \frac{\sum_{q} e_{q}^{2} \Delta \hat{f}_{q / p^{\uparrow}}\left(x, \boldsymbol{k}_{\perp}\right) \otimes \frac{d \hat{\sigma}^{\ell q \rightarrow \ell q}}{d Q^{2}} \otimes D_{h / q}\left(z, p_{\perp}\right)}{2 \sum_{q} e_{q}^{2} f_{q / p}\left(x, k_{\perp}\right) \otimes \frac{d \hat{\sigma}^{\ell q \rightarrow \ell q}}{d Q^{2}} \otimes D_{h / q}\left(z, p_{\perp}\right)},
$$

where $f_{q / p}\left(x, k_{\perp}\right)$ and $D_{h / q}\left(z, p_{\perp}\right)$ are the unpolarized distribution and fragmentation functions, with $\boldsymbol{k}_{\perp}$ and $\boldsymbol{p}_{\perp}$ being, respectively, the transverse momentum of the quark in the proton and of the final hadron $h$ with respect to the fragmenting quark $q$; $\frac{d \hat{\sigma}^{\ell q \rightarrow \ell q}}{d Q^{2}}$ is the partonic cross section corresponding to the underlying elementary process $\ell q \rightarrow \ell q$. The numerator of this azimuthal asymmetry contains the Sivers distribution function [12], $\Delta \hat{f}_{q / p^{\uparrow}}\left(x, \boldsymbol{k}_{\perp}\right)$, related to the number density of unpolarized quarks inside a transversely polarized proton

$$
\Delta \hat{f}_{q / p^{\uparrow}}\left(x, \boldsymbol{k}_{\perp}\right)=\hat{f}_{q / p^{\uparrow}}\left(x, \boldsymbol{k}_{\perp}\right)-\hat{f}_{q / p^{\downarrow}}\left(x, \boldsymbol{k}_{\perp}\right) \equiv \Delta^{N} f_{q / p^{\uparrow}}\left(x, k_{\perp}\right) \boldsymbol{S}_{T} \cdot\left(\hat{\boldsymbol{p}} \times \hat{\boldsymbol{k}}_{\perp}\right) .
$$

DIS 2009 
For the purpose of our fit, we parametrize this function by factorizing the $x$ and $k_{\perp}$ dependences, as follows [2]

$$
\Delta^{N} f_{q / p^{\uparrow}}\left(x, k_{\perp}\right) \propto x^{\alpha_{q}}(1-x)^{\beta_{q}} f_{q / p}(x) h\left(k_{\perp}\right),
$$

where $\alpha_{q}$ and $\beta_{q}$ are free parameters which control the details of the low- $x$ and large- $x$ behaviour of the Sivers function, for each given flavour $q$.

A fit of the available experimental data allowed us the extraction of the Sivers functions, shown in the first panel of Fig. 1, by using the unpolarized distribution and fragmentation function sets (with their appropriate $Q^{2}$ dependence) as given in Refs. [13] and [14] respectively.

The transversity and the Collins functions, which, being chirally odd, can only contribute in pairs
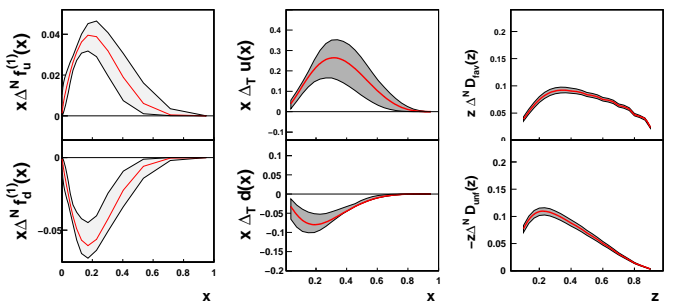

Figure 1: The Sivers, transversity and Collins density functions as determined by fitting SIDIS and $e^{+} e^{-}$data 2, 3.

termined in Ref. [15] and updated in Ref. 3] by performing a simultaneous fit of the Collins azimuthal asymmetry, $A_{U T}^{\text {Collins }}$, measured in SIDIS by HERMES and COMPASS,

$$
A_{U T}^{\text {Collins }} \propto \frac{\sum_{q} e_{q}^{2} h_{1 q}\left(x, \boldsymbol{k}_{\perp}\right) \otimes \frac{d \Delta \hat{\sigma}^{\ell q \rightarrow \ell q}}{d Q^{2}} \otimes \Delta \hat{D}_{h / q^{\uparrow}}\left(z, \boldsymbol{p}_{\perp}\right)}{2 \sum_{q} e_{q}^{2} f_{q / p}\left(x, k_{\perp}\right) \otimes \frac{d \hat{\sigma}^{\ell q \rightarrow \ell q}}{d Q^{2}} \otimes D_{h / q}\left(z, p_{\perp}\right)},
$$

and of the azimuthal correlations $A_{12}$ measured in $e^{+} e^{-} \rightarrow h_{1} h_{2} X$ by the BELLE collaboration [16, 17]. $h_{1 q}\left(x, \boldsymbol{k}_{\perp}\right)$ and $\Delta \hat{D}_{h / q^{\uparrow}}\left(z, \boldsymbol{p}_{\perp}\right)$ are the transversity and Collins functions and $\frac{d \Delta \hat{\sigma}^{\ell q \rightarrow \ell q}}{d Q^{2}}$ is the partonic spin transfer cross section, while $A_{12}$ contains the product of two Collins functions.

The Collins function is related to the number density of unpolarized hadrons $h$ resulting from the fragmentation of a transversely polarized quark:

$$
\Delta \hat{D}_{h / q^{\uparrow}}\left(z, \boldsymbol{p}_{\perp}\right)=\hat{D}_{h / q^{\uparrow}}\left(z, \boldsymbol{p}_{\perp}\right)-\hat{D}_{h / q^{\downarrow}}\left(z, \boldsymbol{p}_{\perp}\right) \equiv \Delta^{N} D_{h / q^{\uparrow}}\left(q, p_{\perp}\right) \boldsymbol{S}_{q} \cdot\left(\hat{\boldsymbol{p}}_{q} \times \hat{\boldsymbol{p}}_{\perp}\right),
$$

where $\boldsymbol{S}_{q}$ and $\boldsymbol{p}_{q}$ are, respectively, the polarization vector and the momentum vector of the fragmenting quark $q$, while $\boldsymbol{p}_{\perp}$ is the intrinsic transverse momentum of the produced hadron with respect to the $\hat{\boldsymbol{p}}_{q}$ direction.

As in the Sivers fit, the transversity (Collins) functions were parametrized so that their $x$ $(z)$ and $k_{\perp}\left(p_{\perp}\right)$ dependences were factorized and their low- $x$ (low- $z$ ) and large- $x$ (large- $z$ ) behaviour controlled by the appropriate $\alpha$ and $\beta$ parameters. The transversity and the Collins functions as determined using this strategy are shown in Fig. 1 One can observe that while the Collins fragmentation functions are rather well constrained thanks to the high statistics of the BELLE data 3, the Sivers and the transversity distributions are affected by much higher uncertainties. Moreover, it is important to notice that the available SIDIS experimental data span a relatively limited range of $x$ values $(x \lesssim 0.3)$ : therefore, the SIDIS data are unable to fix the parameters $\beta$ which control the large- $x$ behaviour of the 
transversity and of the Sivers distribution functions. As a consequence, in our fits the $\beta$ parameters were chosen to be flavor independent. This observation is relevant when turning to polarized proton-proton SSAs [18; in fact, $p p$ experimental data from RHIC cover a range of much larger $x$ values as compared to SIDIS data. When exploring the Sivers and the Collins effects induced in $p p$ processes by the SIDIS extracted functions, this large $x$ uncertainty should be taken into account.

The actual consensus and understanding about the Collins and Sivers functions is that while the former are expected to be universal [19, 20, 21, the latter can be process dependent. In particular the Sivers functions in SIDIS and Drell-Yan processes are expected to be opposite. The situation is much less clear concerning SSAs in $A B \rightarrow h X$ processes in which the only large scale measured is the $P_{T}$ of the final hadron $h$ 22, 23, 24]; even the factorization scheme with transverse momentum dependent distribution and fragmentation functions (TMD factorization) has not been proven in such cases.

We adopt here a pragmatic attitude and explore the possible values of SSAs in $p^{\uparrow} p \rightarrow \pi X$ processes at moderately large $P_{T}$, assuming TMD factorization and universality, that is using the same Sivers and Collins functions as extracted from SIDIS data. A failure to reproduce the experimental results would be a clear indication that these assumptions cannot be valid. The expression of $A_{N}$ in TMD factorization is given by [18:

$$
\begin{aligned}
A_{N} \sim & A_{N}^{\text {Sivers }}+A_{N}^{\text {Collins }} \\
\propto & \frac{\sum_{a, b, c, d} \Delta \hat{f}_{a / p^{\uparrow}}\left(x_{a}, \boldsymbol{k}_{\perp a}\right) \otimes f_{b / p}\left(x_{b}, k_{\perp b}\right) \otimes \frac{d \hat{\sigma}^{a b \rightarrow c d}}{d t} \otimes D_{h / c}\left(z, p_{\perp}\right)}{2 \sum_{a, b, c, d} f_{a / p}\left(x_{a}, k_{\perp a}\right) \otimes f_{b / p}\left(x_{b}, k_{\perp b}\right) \otimes \frac{d \hat{\sigma}^{a b \rightarrow c d}}{d t} \otimes D_{h / c}\left(z, p_{\perp}\right)} \\
+ & \frac{\sum_{a, b, c, d} h_{1}\left(x_{a}, \boldsymbol{k}_{\perp a}\right) \otimes f_{b / p}\left(x_{b}, k_{\perp b}\right) \otimes \frac{d \Delta \hat{\sigma}^{a b \rightarrow c d}}{d t} \otimes \Delta \hat{D}_{h / c^{\uparrow}}\left(z, \boldsymbol{p}_{\perp}\right)}{2 \sum_{a, b, c, d} f_{a / p}\left(x_{a}, k_{\perp a}\right) \otimes f_{b / p}\left(x_{b}, k_{\perp b}\right) \otimes \frac{d \hat{\sigma}^{a b \rightarrow c d}}{d t} \otimes D_{h / c}\left(z, p_{\perp}\right)},
\end{aligned}
$$

where $a, b, c$ and $d$ can be either quarks $q$ or gluons $g$, and all possible pQCD elementary interactions at lowest order contribute. Notice that the Sivers and Collins effects add up in $A_{N}$, and cannot be separated as it is done in SIDIS. Further contributions, proportional to the Boer-Mulders and other TMDs, are negligible, as we have checked numerically [18.

We now apply the quark Sivers, transversity and Collins functions extracted by fitting SIDIS and $e^{+} e^{-}$experimental data to compute $A_{N}$ for $p^{\uparrow} p \rightarrow \pi X$ processes [18] and compare with data [4, 5. As mentioned above, the available SIDIS experimental measurements refer to a limited range of $x$ values $(x \lesssim 0.3)$ : therefore, the SIDIS data are unable to fix precisely the parameters $\beta$ which control the large- $x$ behaviour of the transversity and of the Sivers distribution functions, while the RHIC $p p$ experimental data cover a range of much larger $x$ values. We need to take this large- $x$ behaviour uncertainty into account. We do so by letting the $\beta$ parameters vary; we consider a grid of configurations in which $\beta_{u}$ and $\beta_{d}$, both for the transversity and the Sivers distributions, range from 0 to 4 in steps of 0.5. For each of these configurations, we re-run the SIDIS best fit. Then we select out only the parameter configurations that correspond to a $\chi_{d o f}^{2}$ not larger than about $20 \%$ more of the minimum original value [2, 3]. Finally, we construct a variation band, for both the Sivers and Collins 
contributions to $A_{N}$, given by the convolution of all the curves obtained from the parameter sets we have selected.

Figures 2 and 3 show the results we obtain, from which we can draw a few conclusions. We do not find any clear strong indication of universality breaking effects; on the contrary, given the constraints offered by the presently available SIDIS and $e^{+} e^{-}$experimental data, our results show that there might exist a set of SIDIS extracted Sivers functions which can account for the transverse single spin asymmetry $A_{N}$ for neutral and charged pion production in polarized proton-proton scattering measured by RHIC 4, 5]. Instead, the Collins effect alone, for which universality is usually accepted, only contributes a fraction of the whole $p p$ asymmetries.
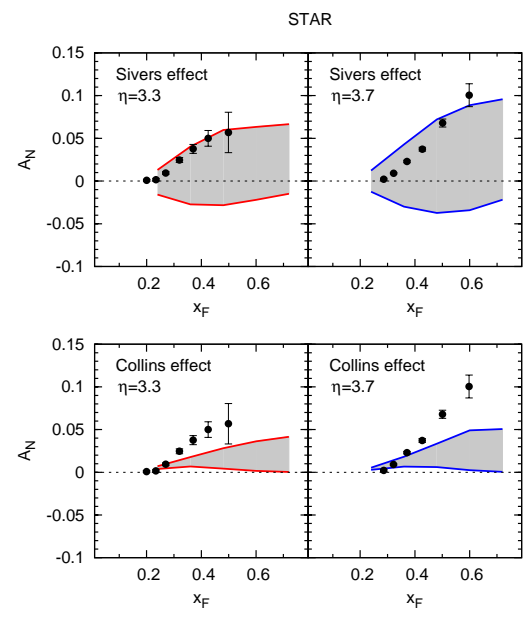

Figure 2: The Sivers (upper panel) and Collins (lower panel) effects, evaluated according to Eq. (6) by using the Sivers, transversity and Collins functions extracted in 2, 3, are compared to the experimental data from STAR [4. The shaded bands are obtained by scanning over the $\beta$ parameters, as described in the text.
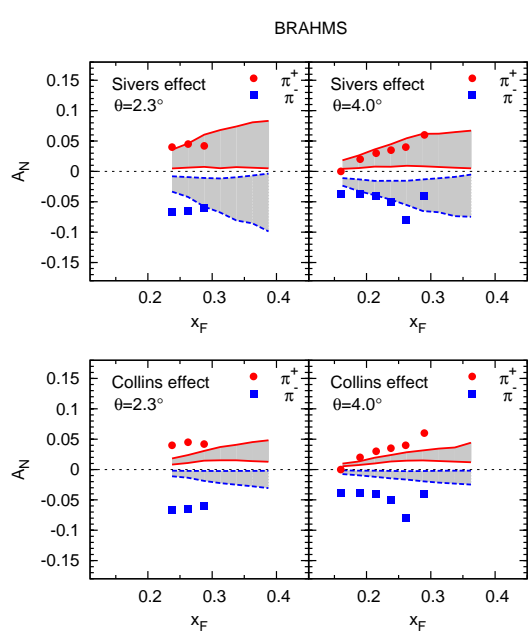

Figure 3: The Sivers (upper panel) and Collins (lower panel) effects, evaluated according to Eq. (6) by using the Sivers, transversity and Collins functions extracted in [2, 3, are compared to the experimental data from BRAHMS [5].
The study of the dependence of these results on the choice of the fragmentation function set is currently under way. There is evidence that this dependence, which is quite mild in SIDIS processes, can be much more pronounced in the case of $p p$ scattering where the cross sections become much more sensitive to the details of the gluon distribution function (recall that there is no glue contribution to SIDIS processes at LO). Moreover the $Q^{2}$ evolution of the Sivers and of the Collins functions are yet unknown. In our fit we assume the same evolution as that of the corresponding unpolarized density functions: the consequences of this simplification have to be analyzed in more details.

These results are entirely phenomenological and preliminary: fur- 
ther studies and data are obviously necessary before one can definitely conclude whether or not the same sets of Sivers and Collins distributions, within a TMD factorized scheme, can explain the SSAs measured in SIDIS and hadronic processes. At the moment we can only conclude that, within the large variation bands, the sum of the (SIDIS extracted) Sivers and Collins contributions could fit the RHIC data on $A_{N}$ at $\sqrt{s}=200 \mathrm{GeV}$.

\section{References}

[1] Talk delivered by M. Boglione. Slides: http: //indico. cern. ch/contributionDisplay . py? contribId=311\&sessionId=4\&conf Id=53294

[2] M. Anselmino et al., Eur. Phys. J. A39, 89 (2009).

[3] M. Anselmino et al., Nucl. Phys. Proc. Suppl. 191, 98 (2009).

[4] B. I. Abelev et al. for the STAR Collaboration, Phys. Rev. Lett. 101, 222001 (2008).

[5] J. H. Lee et al. for the BRAHMS Collaboration, AIP Conf. Proc. 915, (2007).

[6] A. Airapetian et al., Phys. Rev. Lett. 94, 012002 (2005).

[7] M. Diefenthaler, Conference Proceedings of DIS2007, Munich, Germany (2007), pp. 579-582.

[8] V. Y. Alexakhin et al., Phys. Rev. Lett. 94, 202002 (2005).

[9] E. S. Ageev et al, Nucl. Phys. B765, 31 (2007).

[10] A. Martin, Czech. J. Phys. 56, F33 (2006).

[11] M. Alekseev et al., Phys. Lett. B673, 127 (2009).

[12] D. W. Sivers. Phys. Rev. D41, 83 (1990), ibidem D43, 261 (1991).

[13] M. Gluck, E. Reya, and A. Vogt, Eur. Phys. J. C5, 461 (1998).

[14] D. de Florian, R. Sassot, and M. Stratmann, Phys. Rev. D75, 114010 (2007).

[15] M. Anselmino et al., Phys. Rev. D75, 054032 (2007).

[16] K. Abe et al., Phys. Rev. Lett. 96, 232002 (2006).

[17] R. Seidl et al., Phys. Rev. D78, 032011 (2008).

[18] M. Anselmino et al., Phys. Rev. D73, 014020 (2006).

[19] J. C. Collins and A. Metz, Phys. Rev. Lett. 93, 252001 (2004).

[20] F. Yuan and J. Zhou, e-Print: arXiv:0903.4680 [hep-ph].

[21] L. P. Gamberg, A. Mukherjee, and P. J. Mulders, Phys. Rev. D77, 114026 (2008).

[22] A. Bacchetta, C. J. Bomhof, P. J. Mulders, and F. Pijlman, Phys. Rev. D72 , 034030 (2005).

[23] C. J. Bomhof, P. J. Mulders, and F. Pijlman, Eur. Phys. J. C47, 147 (2006).

[24] P. G. Ratcliffe and O. V. Teryaev, e-Print: hep-ph/0703293 